\section{The Bayes Factor, a Suitable Complement beyond Values of $p<0.05$ in Nursing Research and Education}

\author{
Cristian Antony Ramos-Vera' \\ https://orcid.org/0000-0002-3417-5701 (iD)
}

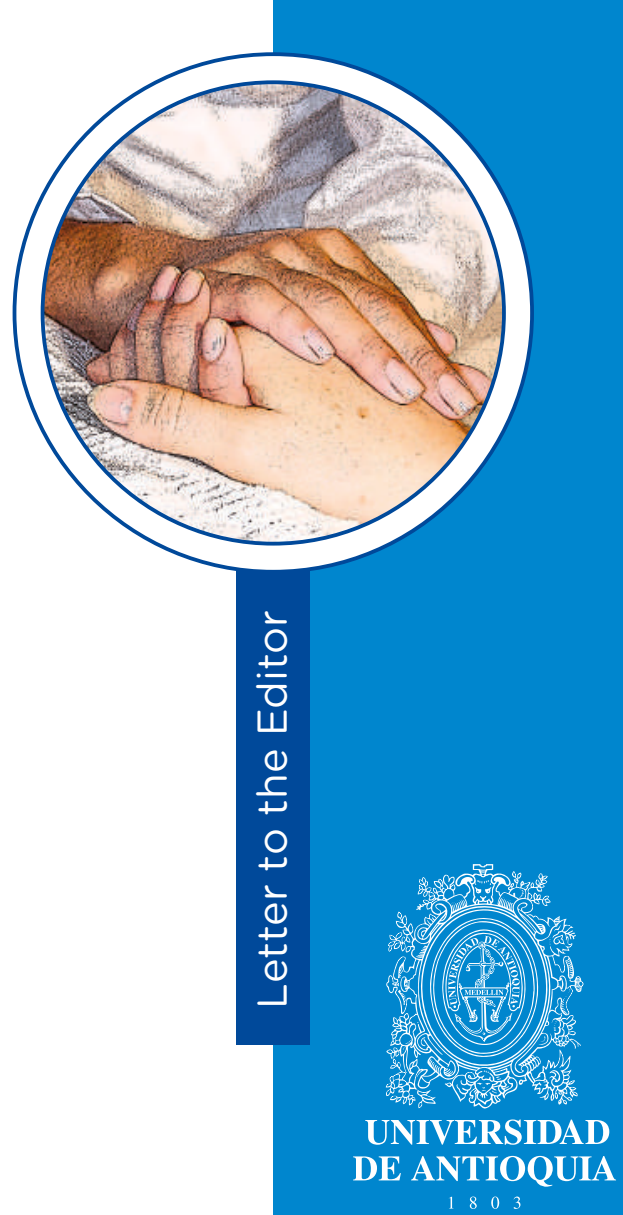

1 Bachelor's degree in psychology. Research Area, Faculty of Health Sciences. Universidad Cesar Vallejo, Lima (Perú). Email: cristony_777@hotmail.com

How to cite this article: Ramos-Vera CA. The Bayes Factor, a Suitable Complement beyond Values of $\mathrm{p}<0.05$ in Nursing Research and Education. Invest. Educ. Enferm. 2021; 39(1):e14

DOI: https://doi.org/10.17533/udea.iee.v39n1e14.

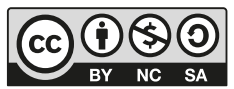

https://creativecommons.org/licenses/by-nc-sa/4.0/ 
Bayesian statistics also permits contrasting hypotheses through probabilities of credibility, being a suitable complement to reinforce statistical significance and, when having frequentist significant findings, it is also a methodological alternative of statistical replication. ${ }^{(3-5)}$ From the Bayesian model, the Bayes factor is the inclusive method of a priori and posteriori credibility to evaluate beyond the level of significance, given that it estimates the degree to which the data support the statistical hypotheses, from Jeffreys' classification scheme for Student's $t$ analysis ${ }^{(5,6)}$ "weak", "moderate", "strong", and "very strong" (Table 1).

When having prior significant results, the only requirement is the t value $(-2.86)$, as well as the sample sizes of both groups (151 and 94) reported by Hoseinabadi et al. ${ }^{(1)}$ Regarding the Bayes factor, it permits inferring two interpretations:
$\mathrm{FB}_{10}$ (in favor of the alternative hypothesis of significant difference) and $\mathrm{BF}_{01}$ (in favor of the null hypothesis from lack of significant difference) and the $95 \%$ confidence interval. ${ }^{(4)}$ Upon the evidence of significant difference, this analysis focuses on estimating the degree of certainty of the alternate hypothesis. The results obtained through the Bayes factor are $\mathrm{BF}_{10}=6.529$ and $\mathrm{BF}_{01}=0.153$, with $95 \% \mathrm{Cl}[-0.388$ to -0.068$]$. These findings report moderate evidence in favor of the statistical hypothesis of significant difference; this may be interpreted in that the alternate hypothesis is six times greater than the nullity of the data and it - in turn - is reduced proportionally for some possible interpretation. Likewise, the parameter is reported of the maximum Bayes factor (maxBF $_{10}$ $=8.444$ ) to determine the stability of the results, which indicates a greater support magnitude to the statistical differences, endorsing the reliability of the findings obtained. ${ }^{(4,7)}$

Table 1. Values of quantifiable interpretation of the Bayes factor*

\begin{tabular}{ccc} 
Value & Interpretation & $\begin{array}{c}\text { Evidence for } \\
\text { the hypothesis }\end{array}$ \\
$>30$ & Very strong & Alternative \\
$10-30$ & Strong & Alternative \\
$3.1-10$ & Moderate & Alternative \\
$1.1-3$ & Weak & Alternative \\
1 & 0 & No evidence available \\
$0.3-0.9$ & Weak & Null \\
$0.3-0.1$ & Moderate & Null \\
$0.1-0.03$ & Strong & Null \\
& Very strong & Null \\
\hline
\end{tabular}

*Elaborated by the author

Likewise, this Bayesian approach is quite useful in other statistical analyses and re-analyses based on the significance value " $p<0.05$ " (correlation, linear regression, logistic regression, ANOVA), which only has a pair of recent guides that permit disseminating the use and interpretation of the Bayes factor beyond nursing research, encompassing its relevance in the health sciences 
in general. ${ }^{(5-7)}$ Furthermore, it permits reinforcing systematic quantitative research that use said statistical tests, thus, providing greater inferential property to meta-analytical studies, and becoming an important methodological contribution inclusively for future articles in this journal.

\section{References}

1. Hoseinabadi TS, Kakhki S, Teimori G, Nayyeri S. Burnout and its influencing factors between frontline nurses and nurses from other wards during the outbreak of Coronavirus Disease (CO-VID-19) in Iran. Invest. Educ. Enferm. 2020; 38(2):e03.

2. Leppink J, O'Sullivan P, Winston K. Evidence against vs. in favour of a null hypothesis. Perspect. Med. Educ. 2017; 6:115-8.

3. Quatto P, Ripamonti E, Marasini D. Best uses of $p$-values and complementary measures in medical research: Recent developments in the frequentist and Bayesian frameworks. J. Biopharm. Stat. 2019; 19:227-46.

4. Ly A, Raj A, Etz A, Gronau QF, Wagenmakers EJ. Bayesian reanalyses from summary statistics: a guide for academic consumers. Adv. Methods Pract. Psychol. Sci. 2018; 1(3):367-74.

5. Kelter R. Bayesian alternatives to null hypothesis significance testing in biomedical research: a non-technical introduction to Bayesian inference with JASP. BMC Med. Res. Methodol. 2020; 20:1-12.

6. Kelter, R. Bayesian and frequentist testing for differences between two groups with parametric and nonparametric two-sample tests. WIREs Comput. Stat. 2020; e15235:393-419.

7. Quintana, DS, Williams DR. Bayesian alternatives for common null-hypothesis significance tests in psychiatry: a non-technical guide using JASP. BMC Psychiatr. 2018; 18(1):178. 